\title{
Test and Analysis of the Mass Properties for the PRANDTL-D Aircraft
}

\author{
Kimberly Callan \\ National Aeronautics and Space Administration Dryden Flight Research Center ${ }^{1}$
}

\begin{abstract}
The main objective of the "PRANDTL-D" project is to obtain the stability and control derivative yawing moment due to aileron deflection, $\mathrm{C}_{\mathrm{n} \delta \mathrm{a}}$. The sign of $\mathrm{C}_{\mathrm{n} \delta \mathrm{a}}$ determines whether the "PRANDTL-D" aircraft experienced proverse or adverse yaw during flight. Proverse yaw, the desirable outcome, occurs when the aircraft yaws in the same direction as the turn due to a novel wing twist and bell-shaped lift distribution. Accurate moments of inertia are essential in order to create a dynamic model of the aircraft and compute the stability and control derivatives, including $\mathrm{C}_{\mathrm{n} \delta \mathrm{a}}$. The robustness of the aircraft simulation also depends on the uncertainty of the mass properties. To obtain the moments of inertia, the aircraft will be hung from two filars and rotated in a bifilar pendulum test. A wireless inertial measurement unit will be used to capture the rotation rate data during testing. This data will be used in a bifilar pendulum simulation to analyze the data and obtain the experimental values. For the tests, our team used Pro Engineer to predict the moments of inertia, designed the test structure and specific testing procedures, and created a Simulink model of the bifilar pendulum test.
\end{abstract}

\section{Background}

The purpose of flight research for the "PRANDTL-D" aircraft (Primary Research for an Aerodynamic Design to Lower Drag) was to obtain the stability and control derivative yawing moment due to aileron deflection, $\mathrm{C}_{\mathrm{n} \delta \mathrm{a}}$, with a positive sign. This derivative represents the adverse yaw experienced by an aircraft. Adverse yaw is the tendency for the aircraft to yaw, or turn in the horizontal plane, in the opposite direction as the intended turn. For past and current aircraft this derivative is found to be negative, showing that these aircraft experience the adverse yaw effect that described. Positive $\mathrm{C}_{\mathrm{n} \delta \mathrm{a}}$ shows the aircraft experienced proverse yaw, a concept that the Dryden Flight Research Center engineers are attempting to prove.

Proverse yaw is the opposite of adverse yaw, when an aircraft yaws in the same direction as the intended turn. Proving proverse yaw can be accomplished in many ways, and the first task that was assigned to my team of interns was to decide how to perform the necessary flight research and prove this concept. The team was presented with a subscale model of a larger flying wing concept. This model had a 12.3 foot wingspan, with a foam interior and carbon fiber overlay. This flying wing utilized a novel wing twist that was computed by my mentor, Albion Bowers, to produce a bell-shaped lift distribution instead of an elliptical distribution, as current wings produce. The production of adverse yaw lies in the lift distribution produced by the wing. An elliptical lift distribution shows that the wing is still producing a large amount of lift at the wingtips. As more drag accompanies the production of more lift, when a wing with an elliptical distribution turns, the outside wing slows itself down due to the amount of drag it is producing at the wingtip. Rudders were created to combat these forces, the effects of adverse yaw. A bellshaped lift distribution, however, produces minimal lift and drag at the wingtips. A wing twist

\footnotetext{
${ }^{1}$ I would like to thank the Wisconsin Space Grant Consortium for funding my internship experience at NASA.
} 
that would produce a bell-shaped lift distribution would allow the outside wing to maintain speed on a turn, creating proverse yaw.

The engineers at the National Aeronautics and Space Administration Dryden Flight Research Center have christened this subscale model "PRANDTL-D," which they have analyzed and calculated to produce a total carbon footprint reduction of approximately $60 \%$. Additional reductions include airframe weight savings of $20-30 \%$ and drag reductions of $8-11 \%$. While many engineers do not believe that the concept of proverse yaw can be proven, the Dryden engineers believe that these calculated reductions are proof in themselves that this idea deserves to be explored. Flying wings similar to "PRANDTL-D" have been flown in the past, however no flight data has ever been recorded.

This is where my intern team had to decide the method in which we would produce flight data. We had to decide what instrumentation would allow us to capture the most accurate data while remaining a logical choice for the aircraft, how to extract the data from these instruments, and how we would analyze this data to obtain the sign of $\mathrm{C}_{\mathrm{n} \delta \mathrm{a}}$. Additionally, we had to select the maneuvers to be performed during flight, bearing in mind the accuracy of our instruments and the ability of our aircraft.

The team decided to break into four subsystems. One subsystem would create a dynamic simulation of the aircraft using Matlab and Simulink; another subsystem, the subsystem I worked with, was in charge of finding the mass properties of the aircraft, including the center of gravity and the moments of inertia; the third subsystem handled the physical integration of the instrumentation on the aircraft; and the last subsystem dealt with day-of-flight procedures, including deciding upon the maneuvers to be performed by the aircraft, the flight cards and briefings, and communications between the team and the RC pilot. The aircraft simulation allowed the team to check that the aircraft would behave in a manner similar to our predictions before flight research was performed on the aircraft. It would also allow us to analyze our data, which we obtained during flight using an unmanned avionics system autopilot called Piccolo II and the appropriate data streaming software (Piccolo Command Center). In order to provide the simulation with a suitable description, or identification, of the "PRANDTL-D" aircraft, the mass properties of the aircraft had to be determined. It is on the mass properties that I focused my research this summer, and it is the mass properties that will be discussed further.

\section{Methods}

As a member of the subsystem in charge of determining the mass properties of the "PRANDTLD" aircraft, it became my objective to experimentally determine the center of gravity and moments of inertia of the aircraft as well as analytically check the experimental moments of inertia. The internship was a ten week experience and my team first received the aircraft during the seventh week. Due to this timing, my subsystem had a lot of time to prepare our procedures but a relatively short amount of time to perform out experiments.

To obtain the center of gravity, my subsystem drew upon previous classroom and project experience. First, we adopted the standard aircraft coordinate system by placing the origin of the coordinate system at the nose of the aircraft. The $\mathrm{x}$ axis then extended positive forward of the nose, the $\mathrm{y}$ axis extended positive over the right wing, and the $\mathrm{z}$ axis extended positive below the 
nose. Next, we decided that we could prepare a relatively simple experiment and obtain accurate results if we could raise the aircraft on a few pinpoints, find the weights of the aircraft at those points, and utilize the concept of a summation of moments. We agreed upon three points, and accomplished this task by raising the nose and wingtips of the aircraft using blocks of wood with nails drilled through them. While the aircraft rested on the points of the nails, the blocks were set on zeroed scales. Then the weights that the scales read and their corresponding distances from the nose were put into Equation 1, below.

Eqn $1 \quad \mathrm{~W}_{\text {aircraft }} \mathrm{d}_{\mathrm{CG}}=\mathrm{w}_{1} \mathrm{~d}_{1}+\mathrm{w}_{2} \mathrm{~d}_{2}+\mathrm{w}_{3} \mathrm{~d}_{3}$.

This method was used to determine the center of gravity along the $\mathrm{x}$ and $\mathrm{y}$ axes. For the $\mathrm{z}$ axis, a slightly different method was employed. For this axis, the original angle that the aircraft made with the ground was measured, along with the distance from the $\mathrm{x}-\mathrm{y}$ center of gravity to a line that was drawn from wingtip to wingtip, or $x_{0}$. The angle the aircraft was tilted was then increased and the new angle and distance measured, $x_{1}$. The difference in the angles was labeled $\theta$, and Equation 2 was then used to find the center of gravity along the $\mathrm{z}$ axis.

Eqn $2 \quad \mathrm{~d}_{\mathrm{CG}}=2 \mathrm{x}_{0} \cos \theta-2 \mathrm{x}_{1}$

Once we had found the center of gravity, our mentors selected a specific location where they then wanted us to place the center of gravity. We used small iron coupons that we inserted into the aircraft's wings to ballast the aircraft. Our mentors asked for the center of gravity to be located 13.24" aft of the nose along the $\mathrm{x}$ axis, as close to the centerline of the aircraft as possible along the $y$ axis, and they were not concerned with the center of gravity along the $\mathrm{z}$ axis.

Once the center of gravity of the aircraft was in the preferred location, the moments of inertia had to be found at that location. It was suggested early on that my subsystem utilize the bifilar pendulum method, a method in which the aircraft is suspended from two cables, or filars, and oscillated around its center of gravity. Before beginning, the subsystem decided upon all the tasks that had to be accomplished before testing. We had to: come up with predictions for the moments of inertia to compare against our experimental results, determine which testing factors would affect our experiment enough to affect our results, design and fabricate the filars and rig that would be used to perform our experiments, and create a Matlab simulation of a bifilar pendulum with which to analyze our data. By spending the appropriate amount of time accomplishing these tasks, testing ran smoothly later.

Obtaining predictions for the moments of inertia that we would experimentally obtain was critical to remain confident with our results from testing. My subsystem used a drafting program, Pro Engineer, with the help of an engineer from the Materials Laboratory, to create a drawing of the entire aircraft, including all of the instrumentation that would be on the aircraft during testing. Using Pro Engineer, we were able to assign the correct material densities to each component of the aircraft. This feature allowed the program to compute all of the moments of inertia and products of inertia of our computer model. We expected some deviation from these predicted results, as the end result of the "PRANDTL-D" aircraft would be slightly different from our ideal computer model. Once we obtained these predictions we were able to employ the 
aircraft simulation subsystem to tell us which moments and products of inertia varied the end result, $\mathrm{C}_{\mathrm{n} \delta \mathrm{a}}$, enough to require being found experimentally. We were informed that we had to find the moments of inertia $I_{x x}$ and $I_{z z}$ and the product of inertia $I_{x z}$ experimentally. The simulation, and therefore the assumed instrumentation of the "PRANDTL-D" aircraft, was robust to the moment of inertia $I_{y y}$ and the rest of the products of inertia. This meant that we did not have to find these inertias experimentally.

Once the moment of inertia and product of inertia predictions were obtained, we had to determine which factors of our experiment might cause the experimental inertia values to vary and if this variance was great enough to cause concern. With the help of one of our mentors, we determined that we had to develop a linearized dynamics model of the bifilar pendulum and calculate the variance of inertia with respect to each parameter. The model of the linearized dynamics model of the bifilar pendulum was created using Equation 3, below.

Eqn $3 \quad I_{b}=\left(\left(m_{\text {comb }}+m_{\text {rigging }}\right) g d^{2}\right) /\left(4 h \omega_{\text {comb }}^{2}\right)-m_{\text {comb }} r_{\text {comb }}^{2}-m_{\text {rigging }} r_{\text {rigging }}^{2}-I_{\text {rigging }}$

The different variables, in the order in which the appear in the equation, include the experimental inertia, the mass of the combined system (aircraft and rigging), the mass of the rigging, gravity, the distance between the filars, the length of the filars, the natural frequency of the combined system, the distance from the center of gravity of the combined system to the axis of rotation, the distance from the center of gravity of the rigging to the axis of rotation, and the inertia of the rigging. Using this equation, the derivative was taken with respect to each parameter to calculate the variance of inertia with respect to each parameter. These variances were then used in Equation 4, below, to calculate the total variance in inertia and to figure out which parameter affects this variance most.

Eqn 4

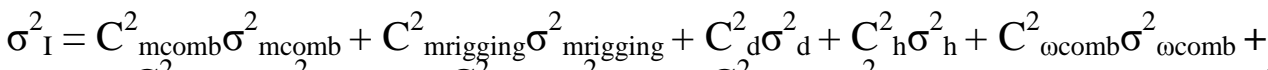

$$
\begin{aligned}
& \mathrm{C}_{\text {wrigging }}^{2} \sigma_{\text {origging }}^{2}+\mathrm{C}_{\text {rcomb }}^{2} \sigma_{\text {rcomb }}^{2}+C_{\text {rrigging }}^{2} \sigma_{\text {rrigging }}^{2}
\end{aligned}
$$

Once the total variance in inertia was found, we applied this procedure to the moment of inertia $\mathrm{I}_{\mathrm{xx}}$. We graphed the total variance in inertia against each parameter and determined that only one graph had a local minimum, and therefore had a specific value to obtain the most accurate data during testing. This parameter was the length of the filars. Graph 1, below, shows the optimal filar length for determining the moment of inertia $\mathrm{I}_{\mathrm{xx}}$ was 1.1 feet.

\section{Graph 1}

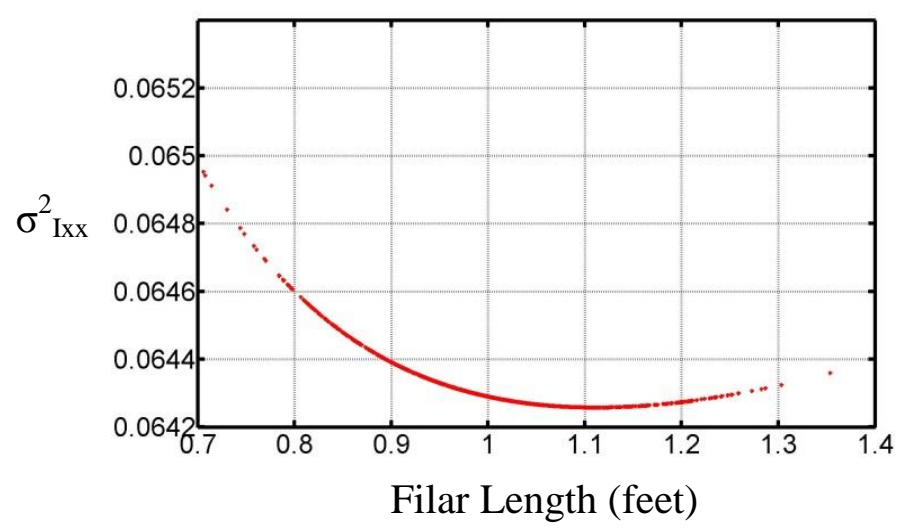


Due to the fact that the predicted values for $I_{x x}$ and $I_{z z}$ were very similar, the optimal filar length for obtaining $\mathrm{I}_{\mathrm{zz}}$ was also 1.1 feet. Soon after we obtained these results, the filars were fabricated. We later realized, however, that due to the testing location we had to use longer filars when experimentally testing for $\mathrm{I}_{\mathrm{xx}}$. We continued to use 1.1 foot filars for the $\mathrm{I}_{\mathrm{zz}}$ experiments, but made additional, 30 inch filars for the $\mathrm{I}_{\mathrm{xx}}$ experiments. This will be discussed in greater detail when I describe the outcome of the experiments.

The next step in preparing for our moments of inertia testing involved assigning personal roles, creating detailed procedures to follow while testing, and determining how we wanted to attach the aircraft to our filars and the filars to the ceiling. After some deliberation, we decided to perform our experiments in Dryden's shuttle hangar, the hangar that was built to accommodate the shuttle in case it was at Dryden while the desert experienced rainfall. Making this decision before typing up our procedures allowed us to keep in mind space and safety considerations. Since my subsystem consisted of three team members, the testing roles we decided upon included a data acquisition engineer, a systems engineer, and an engineering technician. The data acquisition engineer was responsible for inertial measurement unit pre-test setup, collecting all rotation rate data on the laptop, and understanding sensor output. The systems engineer was in charge of directing the team through the testing procedures, making sure all systems were working properly, and checking that all safety guidelines were followed. Finally, the engineering technician made sure to set up test equipment, initiate the tests, and ensure the testing structure was working properly. I was assigned the role of engineering technician. Once these roles were decided, the procedures were drafted. All of the experiments began with attaching the rigging to test structure in the shuttle hangar and to the aircraft. The data acquisition engineer then began data acquisition on the laptop and informed the engineering technician when to initiate each test. The data was collected via a wireless inertial measurement unit that was placed at the center of gravity of the aircraft and attachment plate combination. The attachment plate was fabricated to allow the filars to attach to the aircraft without attaching them to the aircraft itself. Data was collected for thirty oscillations, after which the oscillation of the aircraft was stopped. This procedure was repeated until three successful data sets were acquired. The aircraft was then removed from the rig, leaving all possible connection hardware. The tests were then repeated without the aircraft mounted to the structure to obtain the inertia of the rigging and attachments. These same procedures were followed for the $\mathrm{I}_{\mathrm{zz}}$ experiments apart from the orientation of the aircraft during testing. These procedures were also followed to obtain data for $\mathrm{I}_{\mathrm{xz}}$, although the aircraft was also hung in the $\mathrm{I}_{\mathrm{zz}}$ orientation and the entire procedure was repeated while the aircraft was tilted at angles of $4^{\circ}, 6^{\circ}, 8^{\circ}$, and $10^{\circ}$. Two photographs of testing are shown in Figures 1 and 2 below. 
Figure 1

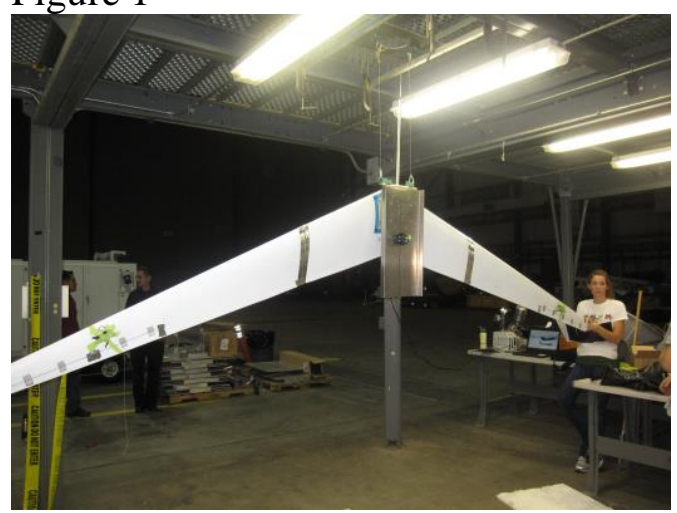

Figure 1 shows the orientation of the aircraft during the $I_{x x}$ experiments.
Figure 2

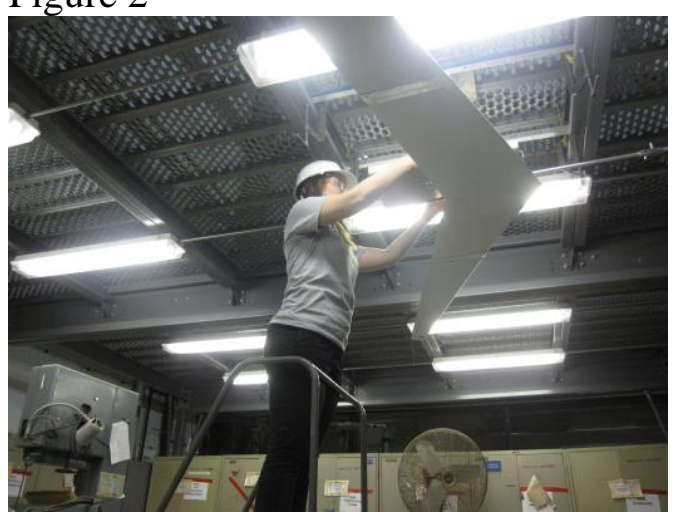

Figure 2 shows the orientation of the aircraft during the $I_{z z}$ experiments. I am initiating the oscillation of the aircraft.

The last piece of preparation that we performed prior to testing included creating a Matlab simulation of a bifilar pendulum with which to analyze our data. The equation of motion of a bifilar pendulum that we input in Matlab, Equation 5, is shown below.

Eqn $5 \quad \ddot{\theta} I+\left(C_{1} \mid(\right.$ theta dot $\left.) \mid+C_{2}\right)($ theta $d o t)+\left(\left(\operatorname{mgd}^{2}\right) /(4 h) \Theta=0\right.$

The simulation was provided with values for theta dot (the rotation rates from the inertial measurement unit) and was able to derive and integrate itself for $\Theta$ and $\ddot{\Theta}$. We also input the mass of the system, gravity, the distance between the filars, and the length of the filars. This left the values of inertia and two nuisance parameters, $\mathrm{C}_{1}$ and $\mathrm{C}_{2}$. The simulation knew what the ideal sine curve would look like under the conditions we set, and then attempted to match the experimental curve that was generated from our rotation rate data to the ideal curve. Once the simulation performed many iterations attempting to match the curves as best as possible, it generated the appropriate inertia and nuisance parameter values. The values of the nuisance parameters were, as their name suggests, not important, but we took note of the inertia values to compare to our predicted values that we generated through Pro Engineer prior to testing.

\section{Results}

There were a few unpredicted complications that occurred during testing. One of these complications concerned the optimal length of the filars that was mentioned earlier. Although the optimal length was 1.1 feet, the orientation of the $\mathrm{I}_{\mathrm{xx}}$ experiments caused a nose attachment on the aircraft to come in contact with the ceiling of the rigging. Since this would greatly interfere with the accuracy of our results, we analyzed the variance graphs that were mentioned previously. We concluded that as the length of the filars increased, the total variance in inertia did not increase enough to cause concern. Since shortening the filars would cause the variance of inertia to greatly increase, we increased the length of the filars to 32 inches for the $I_{x x}$ experiment. Another cause for concern was the air conditioning inside the shuttle hangar itself. We did not predict that the air conditioning would create wind currents strong enough to actually oscillate the aircraft. We contacted the engineer in charge of the shuttle hangar and requested that the air conditioning be turned off for the remainder of the time that we were testing.

As soon as we were presented with a complication that would affect our results we dealt with it so that we would not have to deal with it again during testing. After testing, we also decided to 
check our results once more against the linear equation for inertia. This equation, Equation 6, is shown below.

Eqn $6 \quad \mathrm{I}=\left(\mathrm{mgd}^{2}\right) /\left(4 \mathrm{~h} \omega^{2}\right)$

Our results are shown in Table 1, below.

Table 1

\begin{tabular}{|c|c|c|c|}
\hline Method & $\mathbf{I}_{\mathbf{x x}}$ slug-ft & $\mathbf{I}_{\mathbf{z z}}$ slug-ftt & $\mathbf{I}_{\mathbf{x z}} \mathbf{s l u g - f t}^{\mathbf{2}}$ \\
\hline Predicted & 2.051 & 2.229 & -0.0245 \\
\hline $\begin{array}{c}\text { Experimental } \\
\text { (Linear eqn) }\end{array}$ & 1.695 & 1.599 & -0.048 \\
\hline $\begin{array}{c}\text { Experimental } \\
\text { (w/ simulation) }\end{array}$ & 1.5489 & 1.5068 & -0.0036 \\
\hline
\end{tabular}

\section{Conclusions}

At the end of the internship my subsystem evaluated the effectiveness of the center of gravity and moments of inertia testing preparations. In conclusion, we believe that center of gravity test and procedure were successful. We were able to repeatedly and accurately find the center of gravity each time additional attachments or instrumentation were added to the aircraft. Additionally, we were confident in the bifilar pendulum's ability to yield accurate data for the moments of inertia and product of inertia $\mathrm{I}_{\mathrm{xx}}, \mathrm{I}_{\mathrm{zz}}$, and $\mathrm{I}_{\mathrm{xz}}$. Upon comparing our predicted values and experimental values, we noticed that the experimentally obtained values were lower than the predicted values. This may have occurred as a consequence of Pro Engineer estimating the densities of the materials used in the aircraft. We were confident in our experimental values, however, as they are the same order of magnitude as the predicted values. Also, we expected $\mathrm{I}_{\mathrm{zz}}$ to be larger than $\mathrm{I}_{\mathrm{xx}}$, as our Pro Engineer values predicted. Our values obtained from testing show that this was not the case. Between these values, $\mathrm{I}_{\mathrm{xx}}$ was larger than $\mathrm{I}_{\mathrm{zz}}$. When we checked our results a third time with the linear equation for inertia, these results agreed with our tests and $\mathrm{I}_{\mathrm{xx}}$ was larger than $\mathrm{I}_{\mathrm{zz}}$.

By the end of the internship my team was not able to complete the project as a whole. Flight data still does not exist for this type of aircraft. My mentors at the Dryden Flight Research Center, however, hope to continue this research. 


\section{References}

(1) Chris Regan, Independent Analysis of X-48B Inertia Swing Data Rev. H. 2011

(2) Matt Jardin \& Eric Mueller, Optimized Measurements of UAV Mass Moment of Inertia with a Bifilar Pendulum. 2007

In addition, I would like to recognize and reference Albion Bowers, Oscar Murillo, and Brian Taylor, my three mentors from NASA Dryden Flight Research Center, for their previous work and insight on the project. I would also like to reference my subsystem members, Stephanie Reynolds and Joseph Wagster, the rest of the PRANDTL-D team for their hard work and contributions to the project, and Alex Stuber for his help creating the Pro Engineer model of the PRANDTL-D aircraft. 